\title{
Long non-coding RNA LINC01133 represses KLF2, P21 and E-cadherin transcription through binding with EZH2, LSD1 in non small cell lung cancer
}

\author{
Chongshuang Zang ${ }^{1, *}$, Feng-qi Nie ${ }^{2, *}$, Qian Wang ${ }^{1, *}$, Ming Sun $^{3}$, Wei Li ${ }^{1}$, Jing $\mathrm{He}^{1}$, \\ Meiling Zhang ${ }^{1}$, Kai-hua Lu ${ }^{1}$ \\ ${ }^{1}$ Department of Oncology, First Affiliated Hospital, Nanjing Medical University, Nanjing, People's Republic of China \\ ${ }^{2}$ Department of Oncology, Second Affiliated Hospital, Nanjing Medical University, Nanjing, People's Republic of China \\ ${ }^{3}$ Department of Biochemistry and Molecular Biology, Nanjing Medical University, Nanjing, People's Republic of China \\ *These authors have contributed equally to this work \\ Correspondence to: Kai-hua Lu, e-mail: 13605179453@126.com
}

Keywords: NSCLC, InCRNA, EZH2, LSD1, proliferation

Received: September 16, $2015 \quad$ Accepted: January 20, 2016

Published: January 30, 2016

\section{ABSTRACT}

Long non-coding RNAs are emerging as crucial regulators and prognostic markers in multiple cancers including non small cell lung cancer (NSCLC). In this study, we screened LINCO1133 as a new candidate IncRNA which promotes NSCLC development and progression, in two independent datasets (GSE18842 and GSE19804) from the Gene Expression Omnibus (GEO). LINC01133 is previously found to be over-expressed in lung squamous cell cancer (LSCC) and knockdown its expression inhibits LSCC cells invasion. However, its' molecular mechanism and downstream targets involving in regulation of cancer cells phenotype is not known. Here, we found that LINC01133 expression is up-regulated in NSCLC tissues, and its' over-expression is associated with patients poor prognosis and short survival time. LINC01133 knockdown decreased NSCLC cells proliferation, migration, invasion and induced cell cycle G1/S phase arrest and cell apoptosis. Mechanistic investigations showed that LINC01133 could interact with EZH2, LSD1 and recruit them to KLF2, P21 or E-cadherin promoter regions to repress their transcription. Furthermore, rescue experiments demonstrated that LINC01133 oncogenic function is partly through regulating KLF2. Lastly, we found that there was negative correlation between LINC01133 and KLF2, P21 or E-cadherin in NSCLC. Overall, our findings illuminate how LINC01133 over-expression confers an oncogenic function in NSCLC that may offer a novel therapy target in this disease.

\section{INTRODUCTION}

Non-small cell lung cancer (NSCLC) accounts for $80 \%$ of all lung cancer newly cases, including squamous cell carcinoma (SCC), adenocarcinoma and large cell carcinoma (LCC) [1]. In spite of current advances in the chemotherapy and molecular targeting therapy for NSCLC, the overall 5-year survival rate for NSCLC patients still remains as low as $15 \%$ because patients were at advanced stages when diagnosed $[2,3]$. Lack of biomarkers for early diagnosis and cancer cells metastasis is still one of the most important reasons challenging NSCLC therapy [4]. Therefore, a greater understanding of the molecular mechanisms involved in the development and progression of NSCLC is essential for the developing of specific diagnostic methods and designing of more individualized and effective therapeutic strategies.

In the past decade, accompany with the fast innovation and development of sequencing technique, many important projects have been achieved such as ENCODE and TCGA, and the importance of long noncoding RNAs (lncRNAs) is emerging from the water [5-7]. IncRNAs are new identified member of non-coding family, which are more than $200 \mathrm{nt}$ in length and often expressed in spatial, temporal and tissue-specific pattern 
[6, 8-10]. To date, numerous evidence have revealed that lncRNAs involve in a large range of biological processes including $\mathrm{X}$ chromosome inactivation, reprogramming stem cell pluripotency, muscle cells differentiation and modulation of cell apoptosis and invasion [11-13]. More importantly, the aberrant lncRNAs expression was found to contribute to many human disease including cancers through the regulation of gene expression by chromatin remodeling, DNA or histone protein modification, and function as sponges for microRNAs $[14,15]$.

Recently, IncRNAs disorder have been found to participate in NSCLC development and cancer cells metastasis. For example, lncRNA metastasis-associated lung adenocarcinoma transcript 1 (MALAT1) is a highly conserved nuclear lncRNA, which is up-regulated and could be a predictive marker for metastasis development in lung cancer [16]. Increased HNF1A-AS1 promoted lung adenocarcinoma cells proliferation and metastasis through interacting with DNMT1 and repressing E-cadherin expression [17]. In our previous studies, we found that increased HOTAIR and MVIH promoted NSCLC cells invasion and metastasis via regulating HOXA5, MMP2 and MMP9 expression, and lncRNA ANRIL over-expression increased NSCLC cells proliferation by interacting with EZH2 and recruiting it to KLF2 promoter to repress its transcription [18-20]. These data indicated that lncRNAs play important roles in NSCLC pathogenesis, which could provide new insights into the biology of this disease. Hence, more lncRNA need be identified and investigated.
The IncRNA LINC01133, 1154nt in length, is located in chromosome1q23.2. LINC01133 was recently found to be up-regulated in LSCC tissues and its over-expression is associated with LSCC patients shorter survival time, while knockdown of LINCO1133 inhibited invasion ability of LSCC cell [21]. However, the expression pattern, biological function and underlying mechanism in NSCLC is still completely unknown, which need to be investigated. The aim of this study was to investigate misregulated lncRNAs in NSCLC by analyzing microarray data from GEO datasets, and we focus on the highly up-regulated lncRNA LINC01133. Furthermore, loss and gain of function was performed to investigate the contributions of LINC01133 to NSCLC cell phonotype and the potential molecular mechanism was also investigated. The present study will provide new insights into the biological functions of IncRNA LINC01133 as well as its regulatory mechanisms of targets in NSCLC.

\section{RESULTS}

\section{LINC01133 expression was up-regulated and correlated with poor prognosis of NSCLC}

To investigate aberrant lncRNAs in NSCLC, we firstly analyzed the microarray data from GEO datasets and found that LINC01133 was the highest up-regulated lncRNA in GSE18842 dataset (Figure 1A). In addition, another independent dataset GSE19804 was used to confirmed the LINC01133 expression, which revealed
A GSE18842

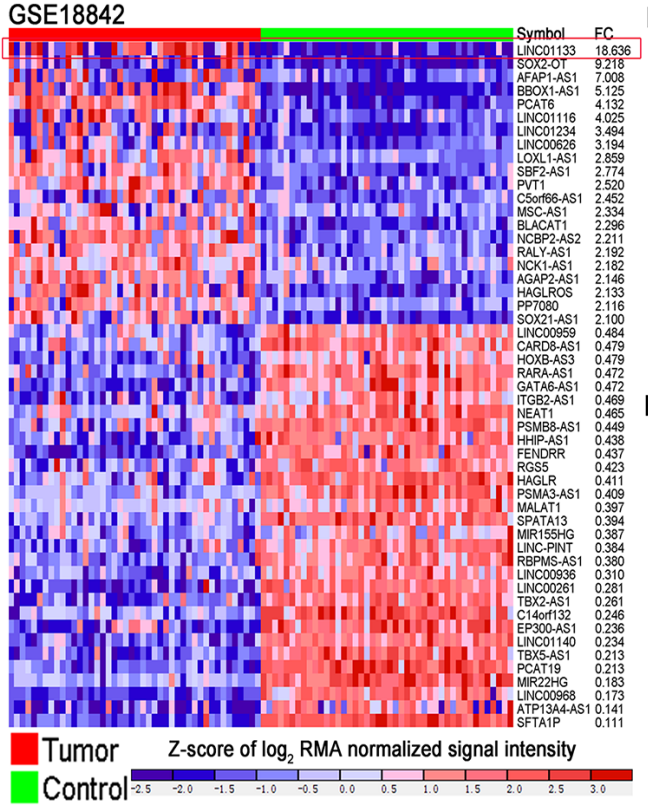

C

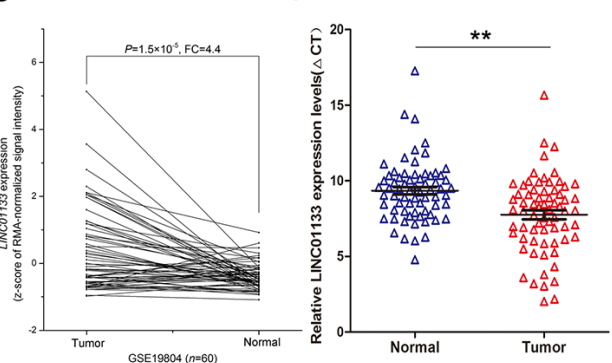

D

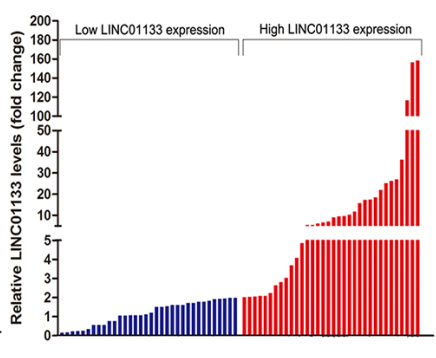

$E$
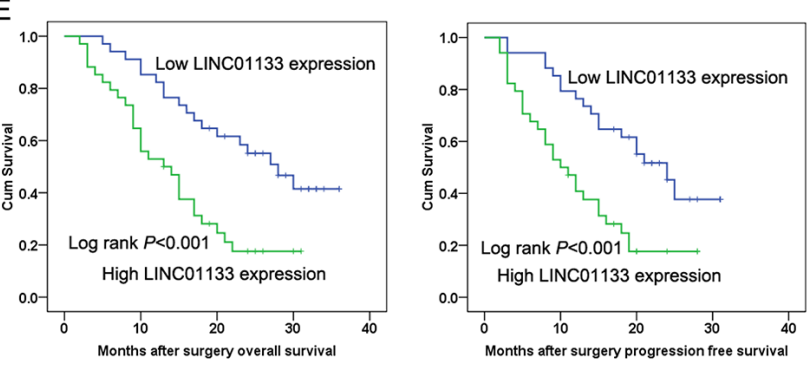

Figure 1: Relative LINC01133 expression in NSCLC tissues and its clinical significance. A, B. Relative expression of LINCO1133 in NSCLC tissues compared with normal tissue was analyzed by using GEO datasets GSE18842 and GSE19804. C. Relative expression of LINCO1133 in NSCLC tissues $(n=68)$ compared with corresponding non-tumor tissues $(n=68)$ was examined by qPCR and normalized to GAPDH expression. Results were presented as the delta CT value. D. LINC01133 expression was classified into two groups. E. Kaplan-Meier overall survival and disease-free survival curves according to LINC01133 expression levels. ${ }^{*} P<0.05,{ }^{* *} P<0.01$. 
that LINC01133 also was up-regulated about 4.4 fold (Figure 1B). Furthermore, LINC01133 expression levels were detected in an cohort of 68 pairs NSCLC tissues and adjacent normal tissues by using qPCR. The results showed that LINC01133 expression was significantly up-regulated (Fold change $>1.5, P<0.01)$ in $74 \%(50 / 68)$ of cancerous tissues compared with normal tissues (Figure 1C). Increased LINC01133 expression levels in NSCLC were significantly correlated with tumor size $(p=0.015)$, advanced pathological stage $(p=0.009)$ and Lymph node metastasis $(p=0.015)$. However, LINC01133 expression was not associated with other parameters such as gender $(p=0.324)$ and age $(p=0.467)$ in NSCLC (Table 1$)$.

Kaplan-Meier survival analysis was conducted to investigate the correlation between LINC01133 expression and NSCLC patients prognosis. According to relative LINC01133 expression in tumor tissues, the 68 NSCLC patients were classified into two groups: the high LINC01133 group ( $n=34$, fold-change $\geq$ mean ratio); and the low LINC01133 group ( $n=34$, fold-change $\geq$ mean ratio) (Figure 1D). The overall survival rate over 3 years for the high LINC01133 group was 21.1\%, and $41.5 \%$ for the low LINC01133 group. Median survival time for the high LINC01133 group was 21months, and 30 months for the low LINC01133 group (Figure 1E). With respect to progression-free survival (PFS), this was $17.6 \%$ for the high LINC01133 group, and $37.7 \%$ for the low LINC01133 group. Median survival time for the high LINC01133 group was 19 months, and 27 months for the low LINC01133 group (Figure 1F).

\section{Modulation of LINC01133 expression in NSCLC cells}

We next performed qPCR analysis to examine the expression of LINC01133 in 8 human NSCLC cell lines, including both adenocarcinoma and squamous carcinoma subtypes (Supplementary Figure S1A). To investigate the functional effects of LINC01133 in NSCLC cells, we modulated its expression through transfection of LINC01133 siRNA or shRNA to knockdown its expression, and LINC01133 vector to over-express its expression. QPCR analysis of LINC01133 levels was performed $48 \mathrm{~h}$ post-transfection, and the results showed that LINC01133 expression was knocked down or overexpressed by si-LINC01133, sh-LINC01133 or pCDNALINC01133 transfection when compared with control cells (Supplementary Figure S1B and S1C).

\section{Knockdown of LINC01133 impaired NSCLC cells proliferation and induced apoptosis}

To assess the roles of LIN01133 in NSCLC, we performed loss- and gain-of-function assays. MTT assays revealed that cell growth was inhibited in A549, H1975 and PC9 cells transfected with si-LINC0113 compared with controls. In contrast, over-expression of LINC01133 could promote SPCA1 cells (with relative low endogenous LINC01133 expression level) proliferation (Figure 2A). Colony formation assay results revealed that clonogenic survival was inhibited following down-regulation of LINC01133 in A549, H1975 and PC9 cells, while LINC01133 over-expression increased SPCA1 cells clone formation ability (Figure 2B and Supplementary Figure S1D). In addition, EdU staining assays also indicated that LINC01133 knockdown decreased NSCLC cells proliferation, while its over-expression increased NSCLC cells proliferation (Figure 2C).

To further examine whether the effect of LINC01133 on proliferation of NSCLC cells reflected cell cycle arrest, cell cycle progression was analyzed by flow cytometry analysis. The results revealed that A549, H1975 and PC9 cells transfected with si-LINC01133 had an obvious cell cycle arrest at the G1/G0 phase and a decreased G2/S phase (Figure 2D and 2E). To determine whether NSCLC cell proliferation was influenced by cell apoptosis, we performed flow cytometry and Tunel staining analysis. The results showed that NSCLC cells transfected with LINC01133 siRNA showed higher apoptotic rate in comparison with control cells (Figure $3 \mathrm{~A}$ and $3 \mathrm{~B}$ ). Moreover, some cell cycle and apoptosis related proteins levels were detected, and the results showed that the levels of Cyclin D1, Cyclin D3, CDK2, CDK4, and CDK6 were decreased in LINC01133 knockdown cells, and Cleaved PARP level was significantly increased (Figure 3C). These data indicate that LINC01133 could promote the proliferation phenotype of NSCLC cells.

\section{Decreased LINC01133 expression inhibits NSCLC cells migration and invasion}

As cancer cells migration and invasion is a significant aspect of cancer progression, we investigate the effect of LINC01133 on NSCLC cells migration and invasion by performing transwell assays. The results showed that decreased LINC01133 expression impeded the NSCLC cells migration and invasion compared with controls (Figure 3D and 3E). These results indicated that knockdown of LINC01133 had tumor-suppressive effects that could inhibit migration and invasion in NSCLC cells.

\section{Down-regulation of LINC01133 inhibits NSCLC cells tumorigenesis in vivo}

To explore whether the level of LINC01133 expression could affect NSCLC cells tumorigenesis, A549 cells stably transfected with sh-LINC01133 or empty vector were inoculated into female nude mice. Eighteen days after the injection, the tumors formed in the sh-LINC01133 group were substantially smaller than those in the control group (Figure 4A). Moreover, the tumor weight at the end of the experiment was markedly 
Table 1: Correlation between LINC01133 expression and clinicopathological characteristics of NSCLC patients

\begin{tabular}{|c|c|c|c|}
\hline \multirow[t]{2}{*}{ Characteristics } & \multicolumn{2}{|c|}{ LINC01133 } & \multirow{2}{*}{$\begin{array}{c}\text { P } \\
\text { Chi-squared test P-value }\end{array}$} \\
\hline & High No. cases (34) & Low No. cases (34) & \\
\hline Age(years) & & & 0.467 \\
\hline$\leq 65$ & 15 & 18 & \\
\hline$>65$ & 19 & 16 & \\
\hline Gender & & & 0.324 \\
\hline Male & 18 & 22 & \\
\hline Female & 16 & 12 & \\
\hline Histological subtype & & & 0.451 \\
\hline Squamous cell carcinoma & 20 & 23 & \\
\hline Adenocarcinoma & 14 & 11 & \\
\hline TNM Stage & & & $0.009^{*}$ \\
\hline $\mathrm{Ia}+\mathrm{Ib}$ & 4 & 14 & \\
\hline $\mathrm{IIa}+\mathrm{IIb}$ & 12 & 12 & \\
\hline IIIa & 18 & 8 & \\
\hline Tumor size & & & $0.015^{*}$ \\
\hline$\leq 5 \mathrm{~cm}$ & 13 & 21 & \\
\hline$>5 \mathrm{~cm}$ & 21 & 13 & \\
\hline Lymph node metastasis & & & 0.015 \\
\hline Negative & 10 & 20 & \\
\hline Positive & 24 & 14 & \\
\hline Smoking History & & & 0.200 \\
\hline Smokers & 20 & 25 & \\
\hline Never Smokers & 14 & 9 & \\
\hline
\end{tabular}

${ }^{*}$ Overall $P<0.05$

lower in the sh-LINC01133 group $(0.458 \pm 0.093 \mathrm{~g})$ compared with the empty vector group $(0.298 \pm 0.073 \mathrm{~g})$ (Figure 4B). QPCR analysis found that the levels of LINC01133 in tumor tissues formed from sh-LINC01133 cells were lower than in tumors formed in the control group (Figure 4C). Tumors formed from sh-LINC01133 transfected A549 cells exhibited decreased positive for Ki67 than those from control cells (Figure 4D). These findings indicate that knockdown of LINC01133 inhibits tumor growth in vivo.

\section{LINC01133 suppressed KLF2, P21 and E-cadherin transcription by interacting with EZH2 and LSD1}

Previously studies have indicated that lncRNAs contribute to cancer cells phenotype through silencing of tumor suppressors or activation of oncogenes via interacting with specific RNA binding proteins. To investigate the potential mechanism of LINC01133 in NSCLC cells, we firstly analysis the distribution of LINC01133 in NSCLC cells and found that LINC01133 mostly located in nucleus (Figure 5A). Then we performed RIP assays to determine the RNA binding proteins (regulate targets at transcriptional levels) which would interact with LINC01133 in NSCLC cells. The results showed that LIN01133 could bind with EZH2 and LSD1 but not other RNA binding proteins in NSCLC cells, and HOTAIR which could also simultaneously bind with EZH2 and LSD1 was used as control ((Figure 5B and Supplementary Figure S1F). In addition, RNA pulldown assays also indicated that LINC01133 could directly binding with EZH2 and LSD1 in A549 cells (Figure 5C).

According to the RIP and RNA pulldown results, we selected some important EZH2 or LSD1 underlying targets and hypothesized that they may also involved in the contributions of LINC01133 to NSCLC development. The 
results of qPCR showed that KLF2, P21 and E-cadherin expression was increased in A549 and PC9 cells with transfection of si-LINC01133; however, there was no significant difference of other genes when knockdown of LINC01133 expression (Figure 5D). Meanwhile, the western blot and immunofluorescence assays showed the same results (Figure $5 \mathrm{E}$ and $5 \mathrm{~F}$ ), which indicated that KLF2, P21 and E-cadherin could be LINC01133 novel targets in NSCLC cells. Furthermore, our qPCR results also showed that inhibition of $\mathrm{EZH} 2$ expression led to increased KLF2, P21 and E-cadherin expression and knockdown of LSD1 up-regulated P21 and KLF2 (Figure 5G). To further investigate whether LINC01133 repressed KLF2, P21 and E-cadherin expression through interacting with EZH2 and LSD1, we performed ChIP analysis and the results showed that EZH2 could directly bind to KLF2, P21 and E-cadherin promoter regions and mediate $\mathrm{H} 3 \mathrm{~K} 27 \mathrm{me} 3$ modification, while LSD1 could bind to P21 and E-cadherin promoter region and mediate $\mathrm{H} 3 \mathrm{~K} 4 \mathrm{me} 2$ demethylation (Figure 5H). However, knockdown of LINC01133 reduced their binding ability (Figure 5I). These data indicated that LINC01133 contributes to NSCLC cells proliferation and apoptosis partly through repressing KLF2 and P21, while regulates cell migration and invasion via silencing E-cadherin expression in NSCLC cells.

To explore entire repertoire of LINC01133-affected genes in NSCLC, we further performed an RNA Seq analysis after silencing of LINC01133 in A549 cells. We found that 307 genes expression was upregulated (log2 fold change $>2$ ), and 149 genes expression was down regulated (Supplementary Figure S2A). Moreover,
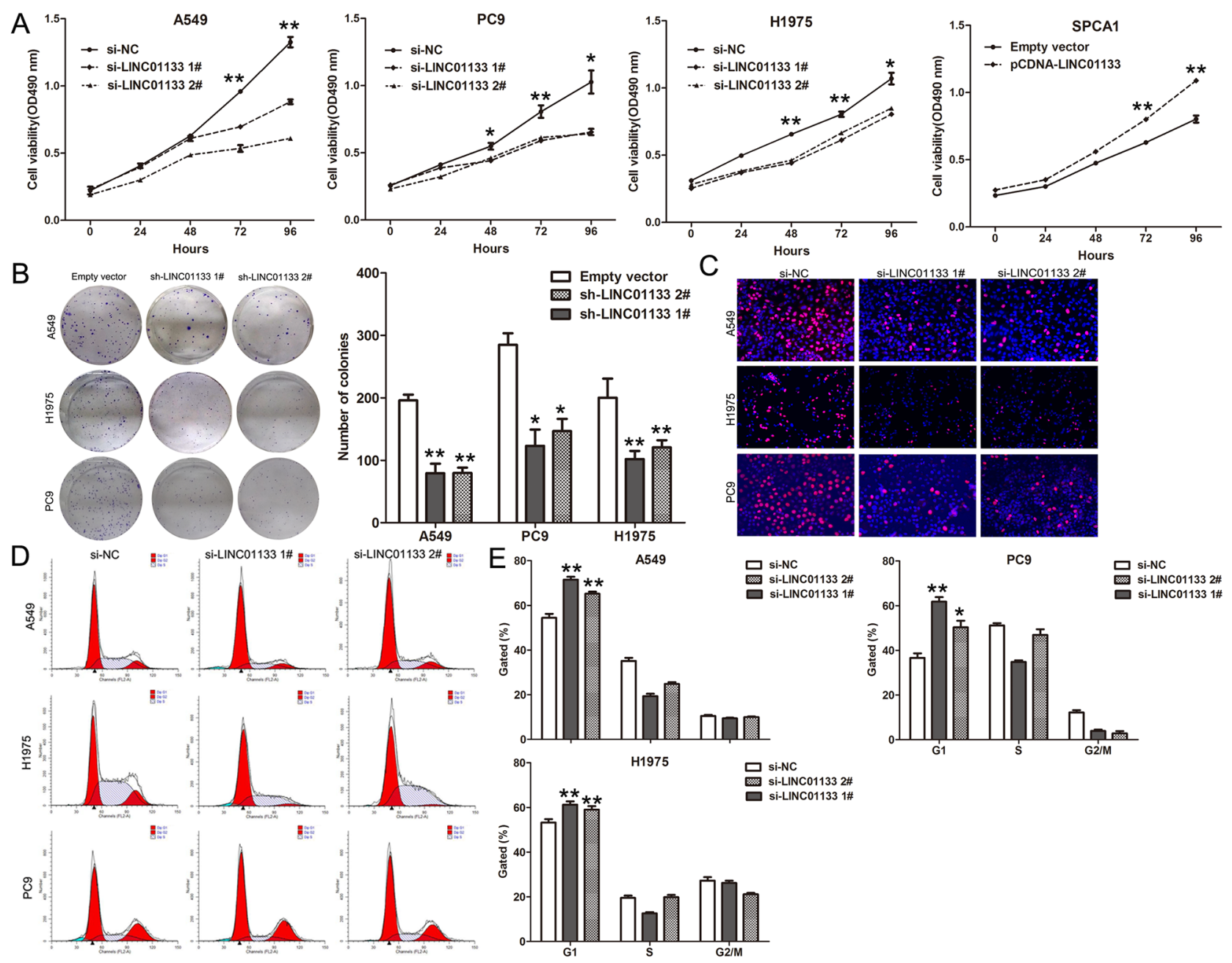

Figure 2: Effects of LINC01133 on NSCLC cell proliferation and cell cycle progression in vitro. A. MTT assays were used to determine the cell viability for si-LINC01133-transfected A549, PC9 and H1975 cells, or pCDNA-LINC01133 transfected SPCA1 cells. Values represented the mean \pm s.d. from three independent experiments. B. Colony-forming assays were conducted to determine the proliferation of si-LINC01133-transfected A549, PC9 and H1975 cells. C. EdU staining assays were conducted to determine the viability of si-LINC01133-transfected A549, PC9 and H1975 cells. D. Flow cytometry assays were performed to analysis the cell cycle progression when NSCLC cells transfected with si-LINC01133. The bar chart represented the percentage of cells in G0/G1, S, or G2/M phase, as indicated. All experiments were performed in biological triplicates with three technical replicates. ${ }^{*} P<0.05,{ }^{* *} P<0.01$. 

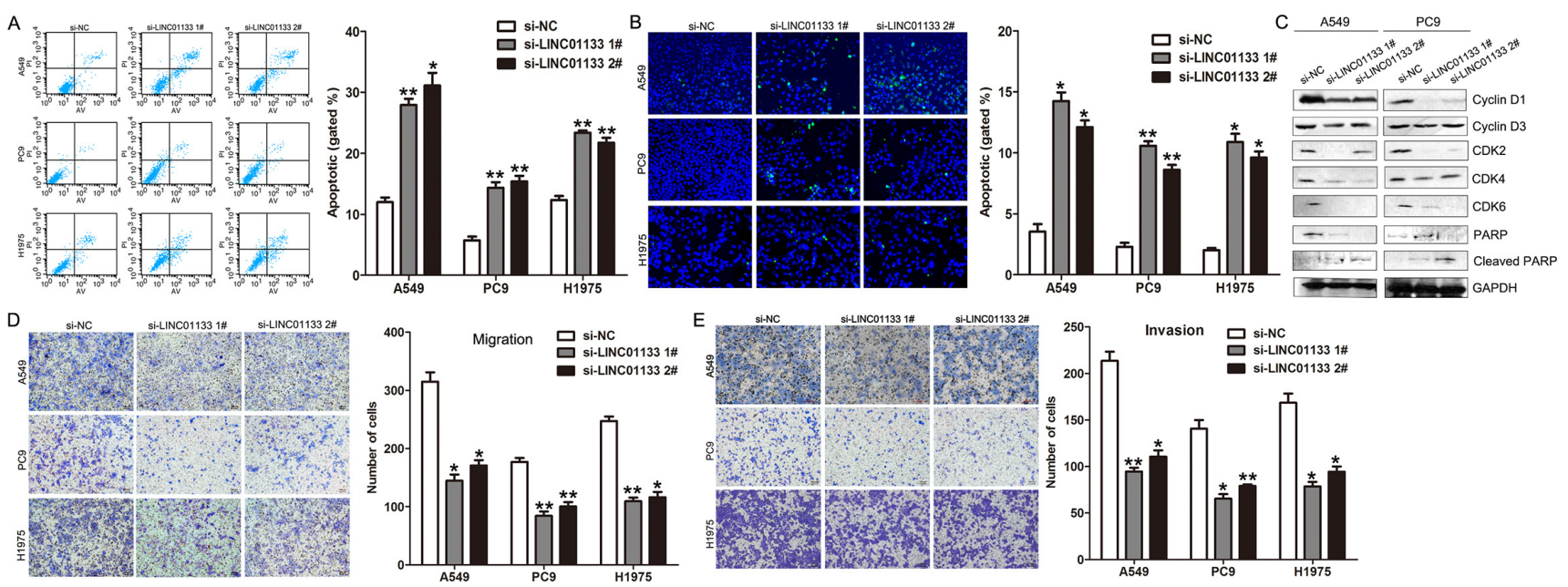

Figure 3: Knockdown of LINC01133 induced NSCLC cell apoptosis and inhibited cell migration and invasion in vitro. A. Flow cytometry assays were performed to analysis the cell apoptotic in si-LINC01133-transfected A549, PC9 and H1975 cells. B. Tunel staining assays were performed to analysis the cell apoptotic when knockdown of LINC01133. C. Effect of knockdown of LINC01133 on cell cycle and apoptosis related proteins expression. D, E. Effect of knockdown of LINC01133 on cell migration and invasion. Data are presented as mean $\pm \mathrm{SD} .{ }^{*} P<0.05,{ }^{* *} P<0.01$.

A
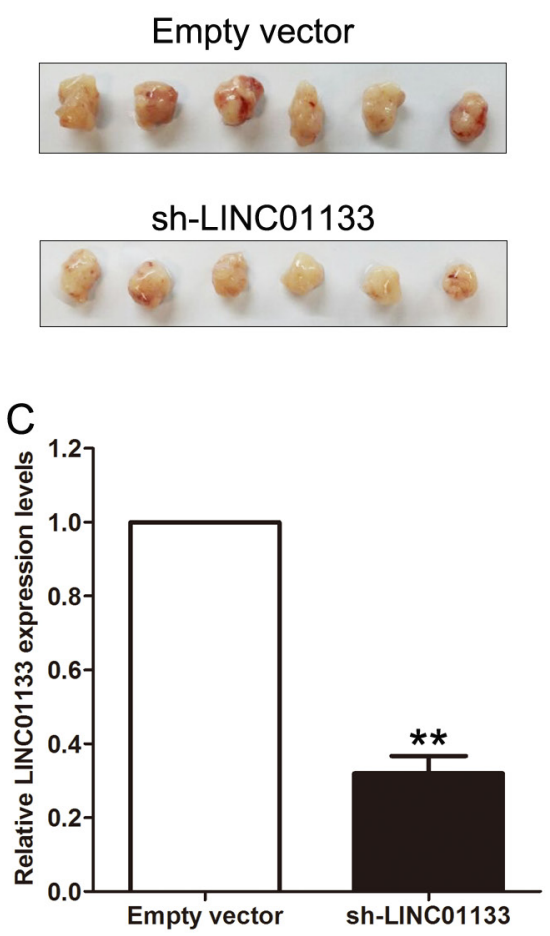
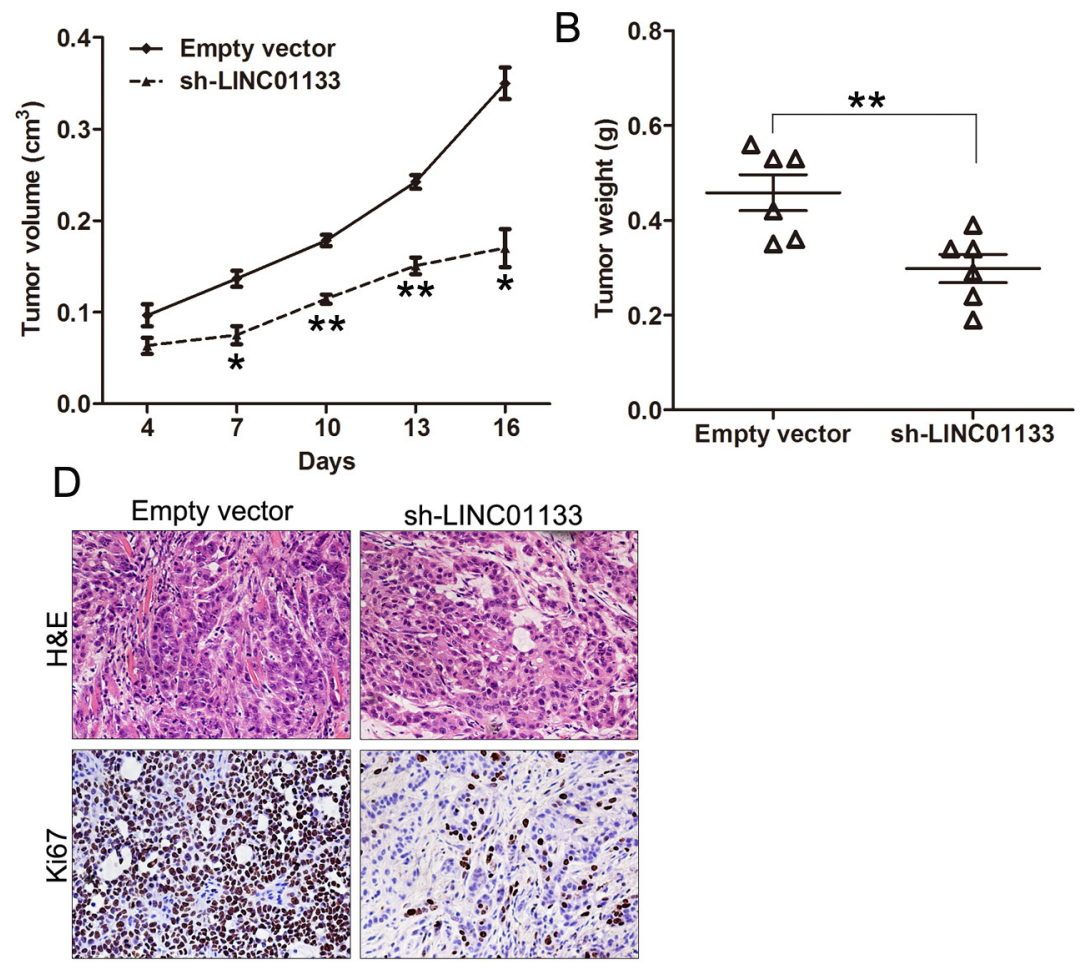

Figure 4: Effect of LINC01133 knockdown on tumor growth in vivo. A. The stable LINC01133 knockdown A549 cells were used for the in vivo study. The nude mice carrying tumors from respective groups were shown and tumor growth curves were measured after the injection of A549 cells. Tumor volume was calculated every 3 days. B. Tumor weights are represented. C. QPCR analysis of LINC01133 expression in tumor tissues formed from A549/sh-LINC01133 and A549/empty vector. D. Tumors developed from shLINC01133 transfected A549 cells showed lower Ki67 protein levels than tumors developed by control cells. Upper: H \& E staining; Lower: immunostaining. ${ }^{*} P<0.05,{ }^{* *} P<0.01$. 

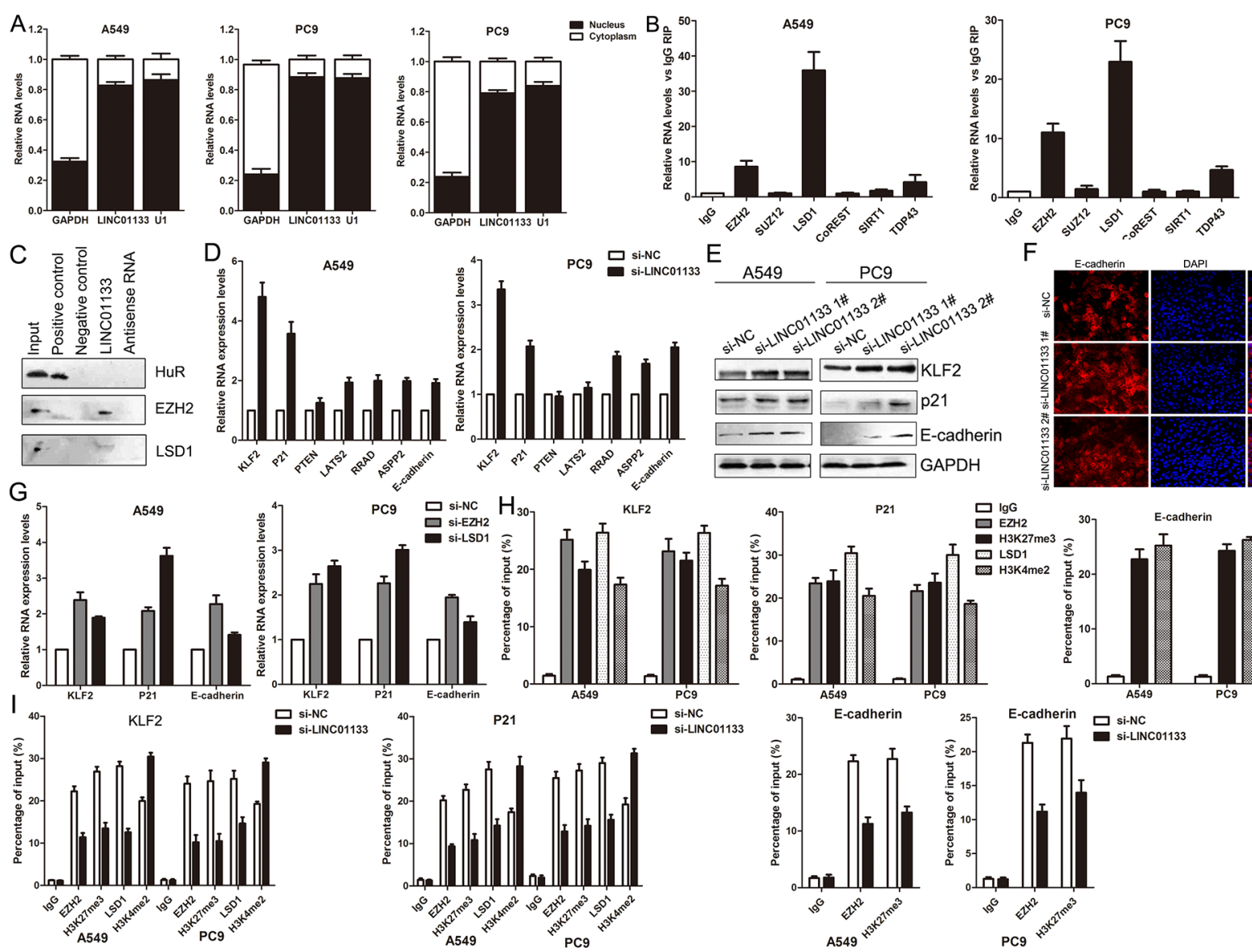

E
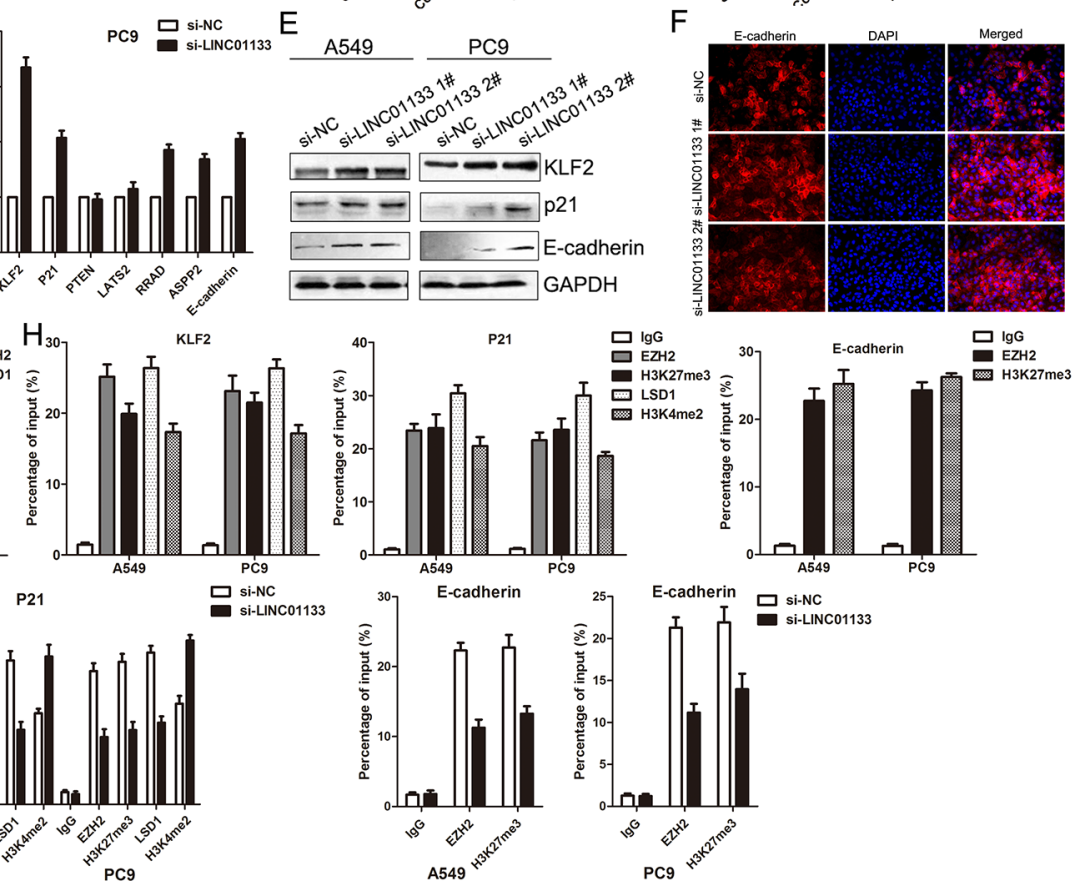

Figure 5: LINC01133 interacted with EZH2 and LSD1, and silence KLF2, P21 and E-cadherin expression. A. Relative LINC01133 levels in cell cytoplasm or Nucleus of NSCLC cell lines were detected by qPCR. B. RNA levels in immunoprecipitates were determined by qPCR. Expression levels of LINC01133 RNA were presented as fold enrichment relative to IgG immunoprecipitates. C. Protein levels in immunoprecipitates were determined by western blot. Expression levels of EZH2 and LSD1 protein were presented. D. The levels of KLF2, P21, PTEN, LATS2, RRAD, ASPP2 and E-cadherin mRNA were determined by qPCR when knockdown of LINC01133. E. The KLF2, P21 and E-cadherin protein levels were determined by western blot in LINC01133 knockdown A549 and PC9 cells. F. The E-cadherin protein levels were determined by immunofluorescence analysis in LINC01133 knockdown A549 cells. G. The KLF2, P21 and E-cadherin expression levels were determined by qPCR when knockdown of EZH2 or LSD1 in A549 and PC9 cells. H, I. ChIP-qPCR of EZH2 and LSD1 occupancy, H3K27me3 and H3K4me2 binding in the KLF2, P21 or E-cadherin promoter in A549 and PC9 cells, and IgG as a negative control. The mean values and s.d. were calculated from triplicates of a representative experiment.

many of these genes are involved in cell growth, cell proliferation and cell death et.al biological process (Supplementary Figure S2B). Next, three of these upregulated genes (EGR1, ATF3 and DDIT3) expression was conformed in A549 cells after knockdown of LINC01133 (Supplementary Figure S2C). These data indicated that there are also additional LINC01133 target genes that are relevant to the observed biological phenotypes

\section{Silence of KLF2 is partly involved in the oncogenic function of LINC01133}

To investigate whether KLF2 is involved in the LINC01133-induced promotion of NSCLC cell proliferation, we performed gain of function assays. As the biological function of KLF2 in PC9 cells has been documented in our previous study, we performed gain of function assays in A549 cells. The results of western blot showed that KLF2 expression was significantly up-regulated in A549 cells transfected with pCDNAKLF2 compared with control cells (Figure 6A). Meanwhile, MTT, colony formation and EdU assays revealed that over-expression of KLF2 could impair A549 cells proliferation (Figure 6B, 6C and 6D). Moreover, flow cytometry analysis indicated that increased KLF2 expression induced A549 cells apoptosis (Figure 6E). Furthermore, to determine whether LINC01133 regulate NSCLC cell proliferation via repressing KLF2 expression, rescue assays were performed. A549 cells were cotransfected with si-LINC01133 and si-KLF2, and this was shown to rescue the increased expression of KLF2 induced by knockdown of LINC01133 (Figure 6F). The results of MTT, colony formation and EdU assays results indicated 

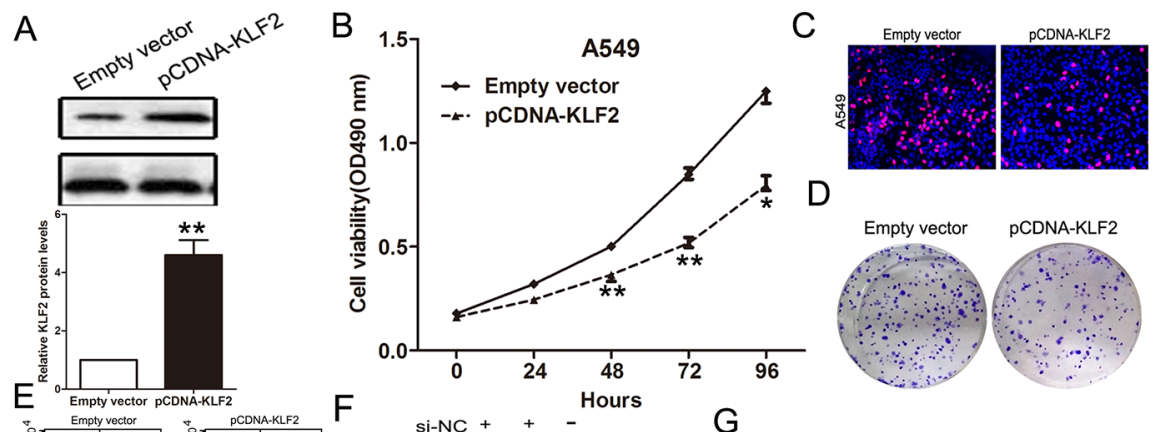

$\mathrm{D}$
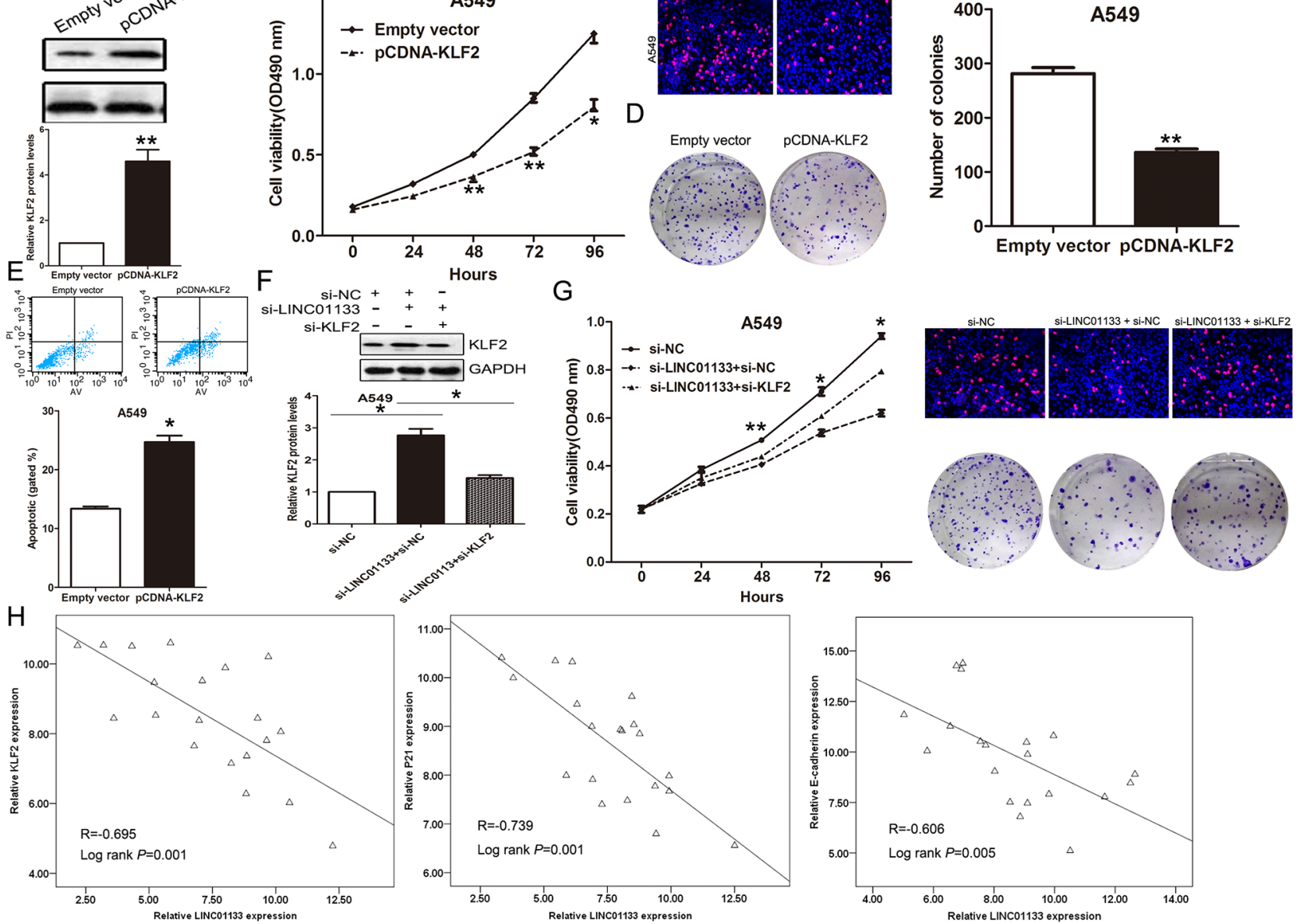

Figure 6: LINC01133 function as oncogene by repressing KLF2 expression in NSCLC cells. A549 cells were transfected with pCDNA-KLF2, or co-transfected with si-LINC01133 and si-KLF2. A. The protein level of KLF2 was detected by western blot. B, C, D. MTT assays, colony-forming and EdU staining assays were used to determine the cell viability. Values represent the mean \pm s.d. from three independent experiments. E. Apoptosis was determined by flow cytometry. F. The protein level of KLF2 was detected by western blot. G. MTT assays, colony-forming and Edu staining assays were used to determine the cell viability. H. Analysis of the relationship between LINC01133 expression and KLF, P21 or E-cadherin expression levels. ${ }^{*} P<0.05$ and ${ }^{* *} P<0.01$.

that co-transfection could partially rescue si-LINC01133impaired proliferation in A549 cells (Figure 6G). Further analysis revealed that LINC01133 expression is inversely correlated with KLF2, P21 and E-cadherin level in 20 paired NSCLC tissues (Figure $6 \mathrm{H}$ ). These data indicate that LINC01133 regulates NSCLC cell proliferation partly through the down-regulation of KLF2 expression.

\section{DISCUSSION}

Recently, more and more studies have revealed that lncRNAs play important roles in multiple cancers development and progression, including NSCLC [22-24]. For example, lncRNA XIST promotes human glioblastoma stem cells proliferation, migration and invasion by sponging miR-152[25]. In addition, lncRNA BANCR exerts tumorsuppressive functions in NSCLC cells, while HOTAIR, MALAT1, LUADT1 and AFAP1-AS1 et.al exert oncogenic function in NSCLC [18, 26-29]. Our previous studies also revealed that lncRNA ANRIL and MVIH function as oncogenes in NSCLC cells, while MEG3 exerts tumorsuppressive function [30]. In this study, our screened another lncRNA LINC01133 that is significantly up-regulated in NSCLC tissues by analyzing GEO datasets, and increased LINC01133 expression may be critically involved in the NSCLC development. Meanwhile, LINC01133 expression also is up-regulated in LSCC (one major histological type of NSCLC), but not in Lung adenocarcinoma (LAD); however, we found that LINC01133 expression is overexpressed in both NSCLC types and correlated with patients poor prognosis, which may due to the specific expression pattern of lncRNA in different population. More important, there is no study that revealed the molecular mechanism and downstream targets of LINC01133 until now. Here, we demonstrated that knockdown of LINC01133 expression exerted tumor-suppressive effects through impairing the cell proliferation, migration and invasion, and inducing cell apoptosis. Furthermore, this study provided evidence for the 
first time that LINC01133 exerted oncogenic functions in human NSCLC cells by interacting with EZH2 and LSD1, and repressing KLF2, P21 and E-cadherin expression.

Generally, IncRNAs regulate downstream targets through interacting with specific RNA binding proteins and leading to inactivation or activation of gene expression via chromosome reprogramming, DNA methylation, histone protein modification or RNA decay $[31,32]$. Our data revealed that LINC01133 could simultaneously interact with EZH2 and LSD1 to repress KLF2, P21 and E-cadherin expression in NSCLC cells. $\mathrm{EZH} 2$ is an core subunit of PRC2 complex that could catalyze the trimethylation of lysine residue 27 of histone 3 (H3K27me3), which is over-expressed in multiple cancers [33]. Highly EZH2 protein expression level is also associated with the early pathogenesis and prognosis of NSCLC patients, and promotes tumor progression via regulating VEGF-A/AKT signaling [34]. In addition, LSD1, one of the enzymatic core of the REST repressor, is the first identified histone demethylase, specifically H3K4me1/2 demethylase [35]. Recent study also revealed that LSD1 is over-expressed in NSCLC and increased LSD1 promoted cell proliferation, migration and invasion [36]. The present study demonstrated that LINC01133 could bind with EZH2 and LSD1, and recruit them to KLF2, P21 or E-cadherin promoter regions to repress their expression in NSCLC cells. These data indicated that LINC01133 may play critical roles in EZH2 and LSD1 mediated repression of tumor suppressors in NSCLC cells.

KLF2, an member of KLF family with Cys2/ His2 zinc-finger domains, is diminished in multiple cancers and possesses tumor-suppressor features such as inhibition of cell proliferation mediated by KRAS [37]. In this study, we also showed that KLF2 can function as tumor suppressor and its' expression could be suppressed by LINC01133 in NSCLC cells. Meanwhile, LINC01133 also silenced E-cadheirn in NSCLC cells, and loss of E-cadherin is an important hallmark of epithelialmesenchymal transition (EMT) process. Numerous of evidence have revealed that EMT is implicated in the promotion of tumor cell invasion and metastasis, and could be a potent mechanism for promoting the detachment of cancer cells from primary tumors [38, 39]. Our data suggest that LINC01133 involved in NSCLC cells migration and invasion might partly through affecting EMT process by repressing E-cadherin expression in NSCLC cells.

In conclusion, we have shown for the first time that LINC0133 expression was up-regulated in NSCLC tissues, suggesting that its up-regulation may be a negative prognostic factor for NSCLC patients. Knockdown of LINC01133 exerted tumor-suppressive functions by reducing cell proliferation, migration and invasion as well as inducing apoptosis in NSCLC cells. Furthermore, LINC01133 mediated the oncogenic effects is partially through its epigenetic silencing of the KLF2, P21 and E-cadherin expression by interacting with EZH2 and LSD1. Our findings further the understanding of NSCLC pathogenesis, and facilitate the development of lncRNAdirected diagnostics and therapeutics against this disease. However, other possible targets and mechanism that underlie regulatory behaviors were not investigated in our study, which still remains to be fully understood and needs to be further investigated.

\section{MATERIALS AND METHODS}

\section{NSCLC samples collection}

68 paired NSCLC and adjacent non-tumor tissues were collected from patients who underwent surgery at Jiangsu Province Hospital between 2010 and 2011, and were diagnosed with NSCLC based on histopathological evaluation. Clinicopathological characteristics, including tumor-node-metastasis (TNM) staging were recorded. There was no local or systemic treatment in these patients before surgery. All collected tissue samples were immediately snap-frozen in liquid nitrogen and stored at $-80^{\circ} \mathrm{C}$ until required. Our study was approved by the Research Ethics Committee of Nanjing Medical University, China. Written informed consent was obtained from all patients.

\section{Cell lines}

Five NSCLC adenocarcinoma cell lines (PC9, SPC-A1, NCI-H1975, H1299, and A549), and three NSCLC squamous carcinomas cell lines (H520, H1703, and SK-MES-1) were purchased from the Institute of Biochemistry and Cell Biology of the Chinese Academy of Sciences (Shanghai, China). A549, H1975, H1299, H1703 and H520 cells were cultured in RPMI 1640; 16HBE, SK-MES-1, PC9 and SPC-A1 cells were cultured in DMEM (GIBCO-BRL) medium supplemented with $10 \%$ fetal bovine serum (FBS), $100 \mathrm{U} / \mathrm{ml}$ penicillin and $100 \mathrm{mg} / \mathrm{ml}$ streptomycin (Invitrogen, Carlsbad, CA, USA) at $37^{\circ} \mathrm{C} / 5 \% \mathrm{CO}_{2}$.

\section{RNA extraction and $q P C R$ assays}

Total RNA was isolated with Trizol reagent (Invitrogen) according to the manufacturer's instructions. Total RNA (1ug) was reverse transcribed in a final volume of $20 \mu \mathrm{l}$ under standard conditions for the PrimeScript RT reagent Kit (TaKaRa, Dalian, China). SYBR Premix Ex Taq (TaKaRa, Dalian, China) was used to determine LINC01133 and targets expression levels, following the manufacturer's instructions. Results were normalized to the expression of glyceraldehyde-3phosphate dehydrogenase $(G A P D H)$. The specific primers are shown in Supplementary Table S1. 


\section{Cell transfection}

Human LINC01133 cDNA and short-hairpin RNA directed against LINC01133was ligated into the pCDNA3.1 and pENTR ${ }^{\text {TM }} \mathrm{H} 1$ vector. Plasmid vectors (pCDNA-LINC01133, pCDNA-KLF2, sh-LINC01133 and empty vector) for transfection were prepared using DNA Midiprep or Midiprep kits (Qiagen, Hilden, Germany), and transfected into SPC-A1, A549, PC9 or H1975 cells. The si-LINC01133 or si-NC were transfected into A549, PC9 or H1975 cells. A549, PC9 or H1975 cells were grown on six-well plates to confluency and transfected using Lipofectamine 2000 (Invitrogen) according to the manufacturer's instructions. At $48 \mathrm{~h}$ post-transfection, cells were harvested for qPCR or western blot analysis.

\section{Cell proliferation assay}

Cell proliferation was monitored using a Cell Proliferation Reagent Kit I (MTT) (Roche Applied Science) and EdU assay kit (Life Technologies Corporation Carlsbad, CA, USA). For MTT assays, cells were seeded into a 96-well plate. Cells per well were added $20 \mu \mathrm{l}$ MTT solution. Plates were incubated for $6 \mathrm{~h}$, and then the absorbance at $490 \mathrm{~nm}$ was measured. For EdU incorporation assay, cells were cultured in 24-well plates. $10 \mu \mathrm{M}$ of EdU was added to each well and cells were cultured for an additional $2 \mathrm{~h}$. Then the cells were fixed with $4 \%$ formaldehyde for $30 \mathrm{~min}$. After washing, EdU can be detected with a Click-iTR EdU Kit for $30 \mathrm{~min}$, and the cells were stained with DAPI for $10 \mathrm{~min}$ and visualized using a fluorescent microscope (Olympus, Tokyo, Japan). The EdU incorporation rate was expressed as the ratio of EdU positive cells to total DAPI positive cells (blue cells), and were counted using Image-Pro Plus (IPP) 6.0 software (Media Cybernetics, Bethesda, MD, USA).

\section{Flow cytometry}

Cells were harvested $48 \mathrm{hr}$ after transfection by trypsinization, and double stained with FITC-Annexin $\mathrm{V}$ and Propidium iodide (PI) using the FITC Annexin V Apoptosis Detection Kit (BD Biosciences). Then, the cells were analyzed with a flow cytometry (FACScan ${ }^{\circledR}$ BD Biosciences) equipped with a CellQuest software (BD Biosciences). Cells for cell cycle analysis were stained with PI using the CycleTEST ${ }^{\text {TM }}$ PLUS DNA Reagent Kit (BD Biosciences) following the protocol and analyzed by FACScan. The percentage of the cells in G0/G1, S, and G2/M phase were counted and compared.

\section{Cell migration and invasion assays}

For cell migration and invasion assays 24-well transwell chambers with $8 \mu \mathrm{m}$ pore size polycarbonate membrane was used (Corning Incorporated, Corning, NY, USA). Cells were seeded on the top side of the membrane pre-coated with Matrigel (BD, Franklin Lakes, NJ, USA) (without Matrigel for cell migration assay).Incubation for $24 \mathrm{~h}$, cells inside the upper chamber were removed with cottons swabs, while cells on the lower membrane surface were fixed and then stained with $0.5 \%$ Crystal violet solution. Five randomly fields were counted randomly in each well.

\section{In vivo tumor formation assay}

Four weeks female athymic BALB/c nude mice were maintained under pathogen-free conditions and manipulated according to protocols approved by the Shanghai Medical Experimental Animal Care Commission. A549 cells were stably transfected with sh-LINC01133 and empty vector and harvested, washed with phosphate-buffered saline (PBS) and re-suspended at a concentration of $1 \times 10^{8}$ cells $/ \mathrm{ml}$. A total of $100 \mu \mathrm{L}$ of suspended cells was subcutaneously injected into each mouse. Tumor growth was examined every 3 days, and tumor volumes were calculated using the equation volume $=$ length $\times$ width $2 / 2$. At 18 days post-injection, mice were euthanized, and the subcutaneous growth of each tumor was examined. The protocol was approved by the Committee on the Ethics of Animal Experiments of the Nanjing medical University.

\section{RNA immunoprecipitation}

For immunoprecipitation (IP) of endogenous PRC2 and LSD1 complexes from whole-cell extracts, cells were lysed. The supernatants were incubated with protein A/G Sepharose beads coated with antibodies that recognized EZH2, SUZ12, LSD1, SNRNP70 or with control IgG (millipore) for $6 \mathrm{hr}$ at $4^{\circ} \mathrm{C}$. After the beads were washed with wash buffer, the complexes were incubated with $0.1 \% \mathrm{SDS} / 0.5 \mathrm{mg} / \mathrm{ml}$ Proteinase K $\left(30 \mathrm{~min}\right.$ at $55^{\circ} \mathrm{C}$ ) to remove proteins, respectively. The isolated from the IP materials was further assessed by qPCR analysis.

\section{RNA pull-down assays}

LINC01133 RNAs were in vitro transcribed using T7 RNA polymerase (Ambio life), which was then purified using the RNeasy Plus Mini Kit (Qiagen) and treated with RNase-free DNase I (Qiagen). Transcribed RNAs were biotin-labeled with the Biotin RNA Labeling Mix (Ambio life). Positive, negative and Biotinylated RNAs were mixed and incubated with A549 cell lysates. Magnetic beads were added to each binding reaction, followed by incubation at room temperature. Then, the beads were washed with washing buffer. The eluted proteins were detected by Western blot analysis.

\section{Chromatin Immunoprecipitation}

A549 and PC9 cells were treated with formaldehyde and incubated for 10 mins to generate DNA- 
protein cross-links. Cell lysates were then sonicated to generate chromatin fragments of 200-300 bp and immunoprecipitated with LSD1, H3K4me2, EZH2 and H3K27me3-specific antibody (Millipore) or IgG as control. Precipitated chromatin DNA was recovered and analyzed by qPCR.

\section{Western blot assay and antibodies}

Cells protein lysates were separated by $10 \%$ SDS-polyacrylamide gel electrophoresis (SDS-PAGE), transferred to $0.22 \mu \mathrm{m} \mathrm{NC} \mathrm{membranes} \mathrm{(Sigma)} \mathrm{and}$ incubated with specific antibodies. ECL chromogenic substrate was used to were quantified by densitometry (Quantity One software; Bio-Rad). GAPDH antibody was used as control, Anti-GAPDH, P21, CDK2, CDK4, CDK6, cyclinD1, cyclinD3, PARP, cleaved PARP and E-cadherin (1:1000) were purchased from Cell Signaling Technology, Inc (CST); Anti-KLF2 were purchased from sigma.

\section{Statistical analysis}

All statistical analyses were performed using SPSS 17.0 software (IBM, Chicago, IL, USA). The significance of differences between groups was estimated by the Student t-test, Wilcoxon test or $\chi^{2}$ test. DFS and OS rates were calculated by the Kaplan-Meier method with the log-rank test applied for comparison. Pearson correlation analyses were used to investigate the correlation between LINC01133 and KLF2, P21 or E-cadherin expressions. Two-sided p-values were calculated, and a probability level of 0.05 was chosen for statistical significance.

\section{ACKNOWLEDGMENTS}

This work was supported by the National Natural Scientific Foundation of China (No.81372397) and the Priority Academic Program Development of Jiangsu Higher Education Institutions (JX10231801) to LKH.

\section{CONFLICTS OF INTEREST}

No potential conflicts of interest were disclosed.

\section{REFERENCES}

1. Siegel R, Naishadham D and Jemal A. Cancer statistics, 2013. CA Cancer J Clin. 2013; 63:11-30.

2. Wistuba, II. Genetics of preneoplasia: lessons from lung cancer. Curr Mol Med. 2007; 7:3-14.

3. Verdecchia A, Francisci S, Brenner H, Gatta G, Micheli A, Mangone L and Kunkler I. Recent cancer survival in Europe: a 2000-02 period analysis of EUROCARE-4 data. Lancet Oncol. 2007; 8:784-796.

4. Klebe S and Henderson DW. Facts and fiction: premalignant lesions of lung tissues. Pathology. 2013; 45:305-315.
5. Djebali S, Davis CA, Merkel A, Dobin A, Lassmann T, Mortazavi A, Tanzer A, Lagarde J, Lin W, Schlesinger F, Xue C, Marinov GK, Khatun J, Williams BA, Zaleski C, Rozowsky J, et al. Landscape of transcription in human cells. Nature. 2012; 489:101-108.

6. Derrien T, Johnson R, Bussotti G, Tanzer A, Djebali S, Tilgner H, Guernec G, Martin D, Merkel A, Knowles DG, Lagarde J, Veeravalli L, Ruan X, Ruan Y, Lassmann T, Carninci $\mathrm{P}$, et al. The GENCODE v7 catalog of human long noncoding RNAs: analysis of their gene structure, evolution, and expression. Genome Res. 2012; 22:1775-1789.

7. Weinstein JN, Collisson EA, Mills GB, Shaw KR, Ozenberger BA, Ellrott K, Shmulevich I, Sander C and Stuart JM. The Cancer Genome Atlas Pan-Cancer analysis project. Nat Genet. 2013; 45:1113-1120.

8. Harrow J, Frankish A, Gonzalez JM, Tapanari E, Diekhans M, Kokocinski F, Aken BL, Barrell D, Zadissa A, Searle S, Barnes I, Bignell A, Boychenko V, Hunt T, Kay M, Mukherjee G, et al. GENCODE: the reference human genome annotation for The ENCODE Project. Genome Res. 2012; 22:1760-1774.

9. Ulitsky I and Bartel DP. lincRNAs: genomics, evolution, and mechanisms. Cell. 2013; 154:26-46.

10. St Laurent G, Wahlestedt $C$ and Kapranov P. The Landscape of long noncoding RNA classification. Trends Genet. 2015; 31:239-251.

11. Fatica A and Bozzoni I. Long non-coding RNAs: new players in cell differentiation and development. Nat Rev Genet. 2014; 15:7-21.

12. Payer B and Lee JT. Coupling of X-chromosome reactivation with the pluripotent stem cell state. RNA Biol. 2014; 11:798-807.

13. Flynn RA and Chang HY. Long noncoding RNAs in cellfate programming and reprogramming. Cell Stem Cell. 2014; 14:752-761.

14. Fatima R, Akhade VS, Pal D and Rao SM. Long noncoding RNAs in development and cancer: potential biomarkers and therapeutic targets. Mol Cell Ther. 2015; 3:5.

15. Shi X, Sun M, Liu H, Yao Y and Song Y. Long non-coding RNAs: a new frontier in the study of human diseases. Cancer Lett. 2013; 339:159-166.

16. Gutschner T, Hammerle M, Eissmann M, Hsu J, Kim Y, Hung G, Revenko A, Arun G, Stentrup M, Gross M, Zornig M, MacLeod AR, Spector DL and Diederichs S. The noncoding RNA MALAT1 is a critical regulator of the metastasis phenotype of lung cancer cells. Cancer Res. 2013; 73:1180-1189.

17. Wu Y, Liu H, Shi X, Yao Y, Yang W and Song Y. The long non-coding RNA HNF1A-AS1 regulates proliferation and metastasis in lung adenocarcinoma. Oncotarget. 2015; 6:9160-9172. doi: 10.18632/oncotarget.3247.

18. Liu XH, Liu ZL, Sun M, Liu J, Wang ZX and De W. The long non-coding RNA HOTAIR indicates a poor prognosis and promotes metastasis in non-small cell lung cancer. BMC Cancer. 2013; 13:464. 
19. Nie FQ, Zhu Q, Xu TP, Zou YF, Xie M, Sun M, Xia R and $\mathrm{Lu} \mathrm{KH}$. Long non-coding RNA MVIH indicates a poor prognosis for non-small cell lung cancer and promotes cell proliferation and invasion. Tumour Biol. 2014; 35:7587-7594.

20. Nie FQ, Sun M, Yang JS, Xie M, Xu TP, Xia R, Liu YW, Liu XH, Zhang EB, Lu KH and Shu YQ. Long noncoding RNA ANRIL promotes non-small cell lung cancer cell proliferation and inhibits apoptosis by silencing KLF2 and P21 expression. Mol Cancer Ther. 2015; 14:268-277.

21. Zhang J, Zhu N and Chen X. A novel long noncoding RNA LINC01133 is upregulated in lung squamous cell cancer and predicts survival. Tumour Biol. 2015; 36:7465-7471.

22. Han D, Wang M, Ma N, Xu Y, Jiang Y and Gao X. Long noncoding RNAs: novel players in colorectal cancer. Cancer Lett. 2015; 361:13-21.

23. Yang X, Xie X, Xiao YF, Xie R, Hu CJ, Tang B, Li BS and Yang SM. The emergence of long non-coding RNAs in the tumorigenesis of hepatocellular carcinoma. Cancer Lett. 2015; 360:119-124.

24. Roth A and Diederichs S. Long Noncoding RNAs in Lung Cancer. Curr Top Microbiol Immunol. Curr Top Microbiol Immunol. 2016; 394:57-110.

25. Yao Y, Ma J, Xue Y, Wang P, Li Z, Liu J, Chen L, Xi Z, Teng H, Wang Z and Liu Y. Knockdown of long noncoding RNA XIST exerts tumor-suppressive functions in human glioblastoma stem cells by up-regulating miR-152. Cancer Lett. 2015; 359:75-86.

26. Sun M, Liu XH, Wang KM, Nie FQ, Kong R, Yang JS, Xia R, Xu TP, Jin FY, Liu ZJ, Chen JF, Zhang EB, De W and Wang ZX. Downregulation of BRAF activated non-coding RNA is associated with poor prognosis for non-small cell lung cancer and promotes metastasis by affecting epithelialmesenchymal transition. Mol Cancer. 2014; 13:68.

27. Guo F, Jiao F, Song Z, Li S, Liu B, Yang H, Zhou Q and Li Z. Regulation of MALAT1 expression by TDP43 controls the migration and invasion of non-small cell lung cancer cells in vitro. Biochem Biophys Res Commun. 2015; 465:293-298.
28. Qiu M, Xu Y, Wang J, Zhang E, Sun M, Zheng Y, Li M, Xia W, Feng D, Yin R and Xu L. A novel lncRNA, LUADT1, promotes lung adenocarcinoma proliferation via the epigenetic suppression of p27. Cell Death Dis. 2015; 6:e1858.

29. Deng J, Liang Y, Liu C, He S and Wang S. The up-regulation of long non-coding RNA AFAP1-AS1 is associated with the poor prognosis of NSCLC patients. Biomed Pharmacother. 2015; 75:8-11.

30. Lu KH, Li W, Liu XH, Sun M, Zhang ML, Wu WQ, Xie WP and Hou YY. Long non-coding RNA MEG3 inhibits NSCLC cells proliferation and induces apoptosis by affecting p53 expression. BMC Cancer. 2013; 13:461.

31. Quinodoz S and Guttman M. Long noncoding RNAs: an emerging link between gene regulation and nuclear organization. Trends Cell Biol. 2014; 24:651-663.

32. Shi X, Sun M, Wu Y, Yao Y, Liu H, Wu G, Yuan D and Song Y. Post-transcriptional regulation of long noncoding RNAs in cancer. Tumour Biol. 2015; 36:503-513.

33. Volkel P, Dupret B, Le Bourhis X and Angrand PO. Diverse involvement of EZH2 in cancer epigenetics. Am J Transl Res. 2015; 7:175-193.

34. Geng J, Li X, Zhou Z, Wu CL, Dai M and Bai X. EZH2 promotes tumor progression via regulating VEGF-A/AKT signaling in non-small cell lung cancer. Cancer Lett. 2015; 359:275-287.

35. Shin J, Ming GL and Song H. Molecular toggle switch of histone demethylase LSD1. Mol Cell. 2015; 57:949-950.

36. Lv T, Yuan D, Miao X, Lv Y, Zhan P, Shen X and Song Y. Over-expression of LSD1 promotes proliferation, migration and invasion in non-small cell lung cancer. PLoS One. 2012; 7:e35065.

37. Kaczynski J, Cook T and Urrutia R. Sp1- and Kruppel-like transcription factors. Genome Biol. 2003; 4:206.

38. Kang Y and Massague J. Epithelial-mesenchymal transitions: twist in development and metastasis. Cell. 2004; 118:277-279.

39. Thiery JP, Acloque H, Huang RY and Nieto MA. Epithelialmesenchymal transitions in development and disease. Cell. 2009; 139:871-890. 